\section{D Imaging and Dentistry \\ From Multiplanar Cephalometry to Guided Navigation in Implantology}

Authors: Giovanna Perrotti / Tiziano Testori / Massimiliano Politi Publisher: Quintessence Publishing

Language: English

ISBN: 978-88-7492-018-1

Edition: 1/e

Publish Year: 2016

Pages: 560, illustrated

Price: $168.00 €$

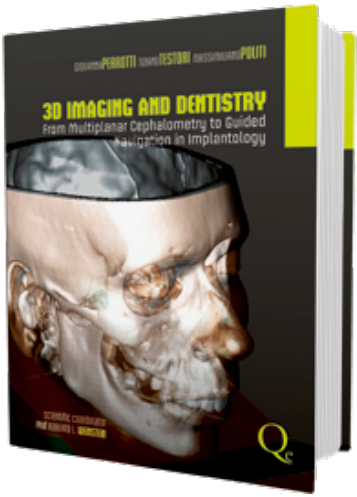

Three-dimensional digital radiology (3D) versus conventional two-dimensional radiology offers the ability to diagnose and plan treatments in dental and maxillofacial surgery, allowing the simulation of surgical procedures.

The book entitled "3D Imaging and Dentistry" is the result of the intellectual effort of a team from the University School of Dentistry at Instituto Ortopedico Galeazzi in Milan. It has a systemic approach and along thirteen chapters presents 3D analysis and CBCT following well-defined internationally acknowledged protocols in different dentistry field.

First the authors present the basic principles for the use of $C B C T$ in dentistry, namely growth and development of facial bone from infancy to senescence, analysis of maxilla and mandible, the concept of cephalometric, soft tissue and skeletal landmarks. The most impressive part of this book is the 3D esthetic analysis of the soft tissues and the diagnostic with Multiplanar, the anatomy and pathology of the TMJ, the 3D analysis of airway spaces, the impactation of permanent maxillary canine and mandibular third molars. The applications of CBCT in periodontology, endodontology, computer-assisted guided surgery and medico-legal issues are also included.

In an illustrative format easy to understand the textbook brings the readers the benefits of using CT/CBCT 3D images for evaluation in many other dental fields such as TMJ, endodontics treatment, and many pathological presentations that could not be visualized with conventional 2D radiography. "3D Imaging and Dentistry" is a book which should be found in the personal library of every practitioner who wishes to provide treatments as accurate as possible to his/her patients.

DOI: 10.25241/stomaeduj.2017.4(3).bookreview.7

Florin-Eugen

Constantinescu

DDS, PhD Student Holistic Dental \& Medical Institute of Bucharest-ROPOSTURO, Bucharest,

Romania
e-mail: drfflorin.constantinescu@gmail.com 